Published in final edited form as:

Am J Gastroenterol. 2018 June ; 113(6): 883-889. doi:10.1038/s41395-018-0104-x.

\title{
Obesity and Response to Infliximab in Patients with Inflammatory Bowel Diseases: Pooled Analysis of Individual Participant Data from Clinical Trials
}

\author{
Siddharth Singh, MD, MS ${ }^{1,2}$, James Proudfoot, MS $^{3}$, Ronghui $\mathrm{Xu}, \mathrm{PhD}^{4}$, William J. \\ Sandborn, MD1 \\ ${ }^{1}$ Division of Gastroenterology, University of California San Diego, La Jolla, California; \\ 2Division of Biomedical Informatics, University of California San Diego, La Jolla, California; \\ ${ }^{3}$ Biostatistics Unit, Altman Clinical and Translational Research Institute; \\ ${ }^{4}$ Department of Family Medicine and Public Health and Department of Mathematics, University of \\ California San Diego, La Jolla, California
}

\section{Abstract}

Aims: To assess whether obesity may affect response to infliximab, we conducted an individual participant data (IPD) pooled analysis using data from clinical trials of infliximab in inflammatory bowel diseases (IBD), using the Yale Open Data Access (YODA) Project.

Methods: We analysed IPD from 4 clinical trials of infliximab in adults with IBD (ACCENT-I, SONIC, ACT-1 and -2). Patients were categorized as obese (body mass index [BMI] $230 \mathrm{~kg} / \mathrm{m}^{2}$ ) vs. non-obese, and by quartiles based on BMI or weight at time of trial entry. Primary outcome was clinical remission (Crohn's disease activity index $[\mathrm{CDAI}]<150$ or pediatric $\mathrm{CDAI}<10$, Mayo Clinic Score <3); secondary outcomes were clinical response and mucosal healing. Multivariable logistic regression analysis was performed, after adjusting for sex, smoking, disease activity, and concomitant prednisone and/or immunomodulators.

Results: We included 1207 infliximab-treated patients (mean age 37y, 51.6\% males, 14\% obese). Obesity was not associated with odds of achieving clinical remission (obese vs. non-obese: adjusted OR, 0.93 [95\% CI, 0.47-1.46]; Q4 vs. Q1: aOR, 0.94 [0.61-1.47], p-value for

\footnotetext{
Corresponding author: Siddharth Singh, MD, MS, Assistant Professor of Medicine, Division of Gastroenterology and Division of Biomedical Informatics, University of California San Diego, 9452 Medical Center Dr., ACTRI 1W501, La Jolla, CA 92093, sis040@ucsd.edu, Phone: 858-246-2352; Fax: 858-657-7259. Author Contribution:

- $\quad$ Study concept and design: SS, WJS

- $\quad$ Acquisition of data: SS, JP

- Analysis and interpretation of data: SS, JP, RX

- $\quad$ Drafting of the manuscript: SS

- Critical revision of the manuscript for important intellectual content: JP, RX, WJS

- Approval of the final manuscript: SS, JP, RX, WJS

- $\quad$ Guarantor of the article: SS
} 
trend=0.97), clinical response ( $\mathrm{Q} 4$ vs. $\mathrm{Q} 1$ : aOR, 0.84 [0.52-1.35], $\mathrm{p}=0.45)$ or mucosal healing (Q4 vs. Q1: aOR, 1.13 [0.55-2.34], $\mathrm{p}=0.95)$. These results were consistent across strata based on disease type (Crohn's disease and ulcerative colitis) and trial design (induction and maintenance therapy).

Conclusions: Based on IPD pooled analysis, obesity is not associated with inferior response to infliximab in patients with IBD. Future studies examining the association between obesity and fixed-dose therapies are warranted.

\section{Keywords}

Open science; posthoc analysis; obesity; complications; anti-TNF

\section{INTRODUCTION}

The global prevalence of obesity is rising, in parallel with the prevalence of inflammatory bowel diseases (IBD).(1) Approximately 15-40\% of IBD patients are obese.(2-4) Obesity has been associated with increased risk of developing diseases such as Crohn's disease (CD) and rheumatoid arthritis (RA).(5) Among patients with IBD, obesity has been variably associated with more severe disease activity, inferior quality of life and higher burden of hospitalization. $(6,7)$ Observational studies in various rheumatic diseases have shown a negative impact of obesity on response to therapy, including tumor necrosis factor-a (TNF) antagonists, both agents that are dosed based on body weight (infliximab), as well as fixeddosing regimens (adalimumab, golimumab, certolizumab pegol or etanercept),(8-11) though these results have inconsistent in patients with IBD. $(12,13)$ This may be attributed to low systemic drug exposure resulting in low trough concentrations in obese individuals (as has been observed in population pharmacokinetic studies), or may be attributed to obesityinduced low-grade inflammation, which can lead to higher systemic inflammatory burden.(4, $14,15)$

Less than $10 \%$ of clinical trials of anti-TNF agents have reported outcomes stratified by body mass index (BMI); where reported, inconsistent and dichotomous weight categories are used, and analyses are not adjusted for potential confounding variables, which limits a comprehensive assessment of impact of obesity on response to therapy.(11) Small, singlecentre observational studies are frequently underpowered and use non-standardized outcome metrics; several have been published only in the abstract form. To overcome limitations of existing studies, and to comprehensively study the association between obesity and response to infliximab in patients with IBD, we conducted a pooled analysis of individual participant level data (IPD) from pivotal trials of infliximab in Crohn's disease (CD) and ulcerative colitis (UC), available through Yale Open Data Access (YODA) Project.(16)

\section{METHODS}

\section{Data Source and Trials}

Clinical trials of infliximab in adult patients with moderate to severe luminal CD or UC were accessed through the YODA project. This pioneering data-sharing model, started in 2011, housed at Yale University, provides access to de-identified IPD data, shared by data 
holders, Johnson\&Johnson, Medtronic, Inc. and SI-BONE, Inc.(16) A detailed research proposal was submitted by the investigators on October 2, 2015 (Protocol \#2015-0612), and after review by the YODA scientific committee, was approved on October 20, 2015, with final data user agreements signed on December 9, 2015. Through this project, we were able to access trials of infliximab in IBD including: ACCENT-I (NCT00207662, Sponsor protocol No., C0168T21), ACCENT-II (NCT00207766, C0168T26) SONIC (NCT00094458, C0168T67), Targan et al (NCT00004941, C0168T20) ACT-1 (NCT00036439, C0168T37), ACT-2 (NCT00096655, C0168T46), UC-SUCCESS (NCT00537316, P04807), REACH (NCT00207675, C0168T47) and an open-label trial of infliximab in UC (NCT00336492, C0168T72). Of these, three trials with insufficient data on drug exposure or weight and outcome assessment (NCT00537316, NCT00004941, NCT00336492), one trial in patients with fistulising CD (NCT00207766) and one trial in pediatric patients (NCT00207675) were excluded.

From these trials, we created a cohort of infliximab-treated patients (with or without concomitant immunomodulators) to study the association between obesity and response to infliximab.

\section{Exposure}

We abstracted data on weight and height (where available) at time of trial entry (screening visit), to calculate body mass index (BMI). Patients within each trial were then divided into obese (BMI $230 \mathrm{~kg} / \mathrm{m}^{2}$ ) vs. non-obese (BMI $<30 \mathrm{~kg} / \mathrm{m}^{2}$ ), and quartiles by BMI (or weight where BMI measures were unavailable), which formed our primary exposure groups.

\section{Outcome}

Primary outcome of interest was achieving clinical remission. This was defined as: Crohn's disease activity index (CDAI <150) (in trials of adults with CD), (17) pediatric CDAI <10 (in pediatric trials of CD), (18) and Mayo Clinic Score [MCS] $<3$ (in trials of adults with UC). (19) Secondary outcomes of interest included: (a) clinical response (decrease in CDAI by $>100$ points [CR-100]; decrease in pediatric CDAI to 11-30 in children; decrease in MCS by $\geq 3$ points and at least $30 \%$ from baseline), and (b) mucosal healing (in patients with CD, absence of ulceration in patients with ulcers present at baseline; MCS endoscopy sub-score of 0 or 1$)$.

Outcomes were abstracted at primary endpoint of the included trials. Trials that reported outcomes for both induction therapy and maintenance were included in the analyses.

\section{Confounding Variables}

We also abstracted data on relevant confounding variables including: age, sex, smoking status (classified as never smokers, prior smokers classified as those who quit $>1$ year prior to trial entry and $<1$ year prior to trial entry, and current smokers), baseline disease activity (CD: severe $>300$, moderate 220-300, mild <220; UC: severe MCS $>9$, moderate 6-9, mild $<6)$ and concomitant use of immunomodulators and/or prednisone. $(17,19)$ Results on disease duration, biochemical parameters including fecal calprotectin and albumin were inconsistently reported in trials, and hence, not included in the multivariate analysis. 


\section{Statistical Analysis}

Baseline characteristics of trials participants were summarized as mean (standard deviation) or medians (range) for continuous variables, and as frequency (\%) for categorical variables, and statistical differences in these characteristics by BMI/weight quartiles were assessed using ANOVA (with two sample t-tests for pairwise comparisons) for continuous variables and the chi-squared test for categorical variables. We compared proportions of patients with desirable outcome (clinical remission, response or mucosal healing) across quartiles of BMI/ weight, across trials, using the chi-square test. Subsequently, we performed multivariable logistic regression analysis to analyze the association between obesity and outcomes, with study as a fixed covariate to account for inter-study differences and with and without adjusting for confounding variables (sex, smoking status, baseline disease activity, concomitant corticosteroids, concomitant immunomodulators). Age was also not reported in the YODA platform for ACT-1 and -2, and hence, was not included in multivariable analysis. Results were reported as odds ratio (OR) and 95\% confidence intervals (CI), using Q1 (or non-obese status) as reference category. A p-value for the linear trend in odds across $\mathrm{BMI} /$ weight quartiles and BMI levels was estimated. Additionally, to evaluate dose-response relationship, we evaluated obesity as a continuous variable reporting risk of outcomes per 1 unit $\left(1 \mathrm{~kg} / \mathrm{m}^{2}\right)$ increase in BMI and per $5 \mathrm{~kg}$ increase in weight.

A priori subgroup analyses were performed based on disease type (CD and UC) and trial design (induction therapy [6-10 weeks] and maintenance trials [26-54 weeks]). All analyses were performed using R (the R Project for Statistical Computing).(20)

\section{RESULTS}

\section{Patient Characteristics in Included Trials}

In 4 trials ( 2 trials in patients with luminal CD, 2 trials in patients with $\mathrm{UC}$ ), we included 1207 patients treated with infliximab. Table 1 lists the main characteristics of all patients, stratified by quartile of BMI or weight at entry to each trial. Median BMI of adult patients was $23.5 \mathrm{~kg} / \mathrm{m}^{2}$ (range, $13.0-49.2 \mathrm{~kg} / \mathrm{m}^{2}$ ). Of note, BMI was reported in two trials of CD, whereas only weight was reported in ACT trials in UC; hence, subgroup analyses were performed using quartiles of BMI (or weight where BMI was not reported). Approximately $32.1 \%$ patients were concomitantly on corticosteroids, and $46.0 \%$ were on immunomodulators. As compared to patients in the $1^{\text {st }}$ quartile, patients in the $4^{\text {th }}$ quartile of BMI/weight were older (Q4 vs. Q1: 41.0y vs. 32.0y, p<0.01), were more likely to be males (57.8\% vs. 38.0\%, p<0.01), had lower baseline disease activity (CDAI: 265 vs. 290, p<0.01; no difference in MCS), and were more likely to be on concomitant prednisone $(40.2 \% \mathrm{vs}$. $27.7 \%, \mathrm{p}<0.01)$ at time of cohort entry.

\section{Obesity and Response to Infliximab}

Clinical Remission: Obesity was not associated with the odds of achieving clinical remission. There was no significant difference in the proportion of infliximab-treated patients achieving clinical remission based on obesity status (obese vs. non-obese: $54.4 \%$ vs. 55.0\%; OR, 0.92 [95\% CI, 0.58-1.47], p=0.73) or by quartile analysis based on baseline BMI or weight (Q4 vs. Q1 - 49.1\% vs. 47.6\%; OR, 1.01 [0.71-1.45], p-value for linear 
trend=0.75). Results were unchanged after adjusting for key covariates (aOR, 0.94 [0.601.47], $\mathrm{p}=0.97$ ) (Figure 1, Table 2, eTable 1). When examining obesity as a continuous variable, there was no association between BMI (per $1 \mathrm{~kg} / \mathrm{m}^{2}$ increase in BMI: aOR, 0.99 [0.96-1.03]) (eTable 2) or weight (per 5kg increase in weight: aOR, 1.00 [0.94-1.05) and achievement of clinical remission (eTable 3). On analysis of each trial separately, obesity was associated with lower risk of achieving clinical remission only in ACT-2 (aOR, 0.25 [0.08-0.77], p=0.04) (eTables 4-7).

Clinical Response and Mucosal Healing: Obesity was not associated with risk of achieving clinical response or mucosal healing. After adjusting for covariates, infliximabtreated patients in the highest quartile of BMI were no less likely to achieve clinical response (aOR, 0.90 [0.55-1.49], $\mathrm{p}=0.66$ ) or mucosal healing (aOR, 1.13 [0.55-2.34], $\mathrm{p}=0.95$ )

(Figure 1, Table 2, eTable 1). Overall results were similar on analysis of BMI and weight as continuous variables (eTable 2 and 3), and across individual trials (eTables 4-7).

\section{Subgroup Analysis}

IBD type: Overall results were comparable on analyses stratified by disease type. Obesity was not significantly associated with risk of achieving clinical remission in infliximabtreated patients with $\mathrm{CD}$ (Q4 vs. Q1: aOR, 0.97 [0.56-1.68], $\mathrm{p}=0.87$ ) or $\mathrm{UC}(\mathrm{aOR}, 0.86$ [0.38-1.92], $\mathrm{p}=0.98$ ); of note, trials of UC only reported weight and not BMI (Table 3). Similar results were observed for outcomes of clinical response and mucosal healing, and on per unit analysis (eTable 2 and 3).

Trial design: In trials of induction therapy, there was no difference in risk of achieving clinical remission (aOR, 1.34 [0.83-2.16], p-value for linear trend=0.07), clinical response or mucosal healing based on baseline BMI or weight (Table 4). In trials of maintenance therapy, obesity was not associated with risk of achieving clinical remission in infliximabtreated patients (aOR, 0.92 [0.56-1.51], p=0.37) (Table 4).

\section{DISCUSSION}

In this pooled analyses of pivotal clinical trials of infliximab in 1207 patients with IBD, we observed that obesity does not significantly modify response to infliximab. These results were stable in patients with $\mathrm{CD}$ and $\mathrm{UC}$, and in trials of induction and maintenance therapy. These findings suggest that in patients with IBD treated with an intravenous anti-TNF agent administered in a weight-based dosing regimen, obesity may not significantly influence short- to medium-term clinical outcomes. We are unable to comment on the potential impact of obesity on other biologic agents that are administered in a fixed dose, regardless of body weight.

Prior small observational studies in patients with IBD have shown conflicting results on how obesity may impact response to anti-TNF therapy. In a single-center, retrospective cohort study, Bhalme et al observed, in adalimumab-treated, but not in infliximab-treated patients, higher likelihood of dose escalation in obese patients than in non-obese (BMI $>35 \mathrm{~kg} / \mathrm{m}^{2}$ vs. BMI $<25 \mathrm{~kg} / \mathrm{m}^{2}: 40 \%$ vs. $20 \%$ ).(12) In contrast, in another retrospective cohort study of 124 patients with IBD, obese patients treated with infliximab were 3-9 times more likely to have 
an IBD flare and require biologic dose escalation than normal weight.(13) Each unit increase in BMI was associated a 6\% higher likelihood of CD flare (HR, 1.06; 95\% CI, 1.02-1.11), and $30 \%$ higher likelihood of UC flare (HR, 1.30; 95\% CI, 1.07-1.58). However, these uncontrolled observational studies used non-standard outcome measures and were unable to adequately control for potential confounding variables. Moreover, in real-world studies, it is probable that obese patients treated with infliximab may not receive optimal weightappropriate therapy. Seminerio and colleagues observed that the average dose of infliximab in patients with class III obesity was $\sim 4 \mathrm{mg} / \mathrm{kg}$, compared to $7.9 \mathrm{mg} / \mathrm{kg}$ in normal BMI and $6.4 \mathrm{mg} / \mathrm{kg}$ body weight in overweight patients.(7) By using a more robust study design, including individual participant level data from clinical trials, with validated disease-specific outcomes, adequate drug exposure and adjusting for key confounding variables, we were able to demonstrate that obesity may not be an important effect modifiers in infliximab treated patients with IBD.

In contrast to IBD, data from prospective cohort studies in rheumatic diseases have more consistently suggested that obesity may negatively impact outcomes in infliximab-treated patients. In a prospective cohort of 89 patients with rheumatoid arthritis treated with infliximab, obese patients had lower rates of clinical response (measured using Disease Activity Score in 28 joints) as compared to non-obese patients, even after adjustment for baseline disease activity and anti-citrullinated protein antibody status (BMI $>30 \mathrm{~kg} / \mathrm{m}^{2} \mathrm{vs}$. $20-30 \mathrm{~kg} / \mathrm{m}^{2}$ vs. $<20 \mathrm{~kg} / \mathrm{m}^{2}: 50 \%$ vs. $75 \%$ vs. $84 \%$ ).(10) Similarly, in 155 infliximab-treated patients with ankylosing spondylitis, Ottaviani and colleagues observed that only $26.5 \%$ of obese patients achieved clinical response, as compared to $77.6 \%$ normal BMI and $48.9 \%$ overweight patients.(21) These differences may be related to differences in pathogenesis, drug dosing and impact of severe disease in patients with IBD and rheumatic diseases. It is likely that in IBD patients, local mesenteric fat plays a more important role than systemic obesity, in contrast to other rheumatic diseases.(22) Dose of infliximab approved in patients with IBD is higher than that for other rheumatic diseases, which may overcome potentially detrimental effects of obesity. Finally, differential effect of confounding by disease severity in IBD and rheumatic diseases may also explain this finding - severe IBD is likely to result in weight loss and misclassification of obesity, whereas, severe rheumatic diseases would likely impact physical activity, promote sedentary lifestyle and contribute to obesity.

There are several limitations inherent to our study. First, though we pooled IPD data from trials, there were intrinsic differences in trial participants which may not have been adequately accounted for despite adjustment. However, our analyses were stable on multiple subgroup and sensitivity analyses, and also on analysis if each trial in isolation. Second, some trials reported only weight and hence, we were unable to consistently categorize exposure based on World Health Organization categories of obesity. Third, we were unable to study impact of obesity on trough concentrations of infliximab or need for dose escalation, and hence, we are unable to comment whether obesity may influence systemic drug exposure and clearance in infliximab-treated patients. Since most trials were limited to 1 year, long-term impact of obesity potentially mediated by sub-optimal drug concentrations cannot be ascertained. Fourth, data on weight was collected at time of cohort entry; hence, patients with recent weight changes prior to trial entry could not be ascertained which may have resulted in misclassification of some patients. However, since trials do not enroll very 
sick patients who may have excessive amount of weight loss, the likely impact of such misclassification is low.

In conclusion, obesity does not significantly influence response to infliximab in patients with IBD, based on a pooled analysis of IPD from clinical trials. It is unknown whether obesity may influence response to fixed-dose therapies in patients with IBD. Post-hoc analyses of RCTs with individual participant level data are warranted to ascertain this association.

\section{Supplementary Material}

Refer to Web version on PubMed Central for supplementary material.

\section{Funding:}

This project is supported by the American College of Gastroenterology and the Crohn's and Colitis Foundation. The project was also partially supported by the National Institutes of Health, Grant UL1TR001442. The content is solely the responsibility of the authors and does not necessarily represent the official views of the NIH.

Disclosures: Dr. Singh receives research support from Pfizer. Dr. Sandborn reports grant support and consulting fees from Pfizer, Prometheus Laboratories, AbbVie, Boehringer Ingelheim, Takeda, Atlantic Pharmaceuticals, Janssen, Bristol-Myers Squibb, Genentech, and Nutrition Science Partners, and personal fees from Kyowa Hakko Kirin, Millennium Pharmaceuticals, Celgene Cellular Therapeutics, Santarus, Salix Pharmaceuticals, Catabasis Pharmaceuticals, Vertex Pharmaceuticals, Warner Chilcott, Gilead Sciences, Cosmo Pharmaceuticals, Ferring Pharmaceuticals, Sigmoid Biotechnologies, Tillotts Pharma, AM-Pharma BV, Dr. August Wolff, Avaxia Biologics, Zyngenia, Ironwood Pharmaceuticals, Index Pharmaceuticals, Nestle, Lexicon Pharmaceuticals, UCB Pharma, Orexigen, Luitpold Pharmaceuticals, Baxter Healthcare, Ferring Research Institute, Amgen, Novo Nordisk, Mesoblast Inc., Shire, Ardelyx Inc., Actavis, Seattle Genetics, MedImmune (AstraZeneca), Actogenix NV, Lipid Therapeutics Gmbh, Eisai, Qu Biologics, Toray Industries Inc., Teva Pharmaceuticals, Eli Lilly, Chiasma, TiGenix, Adherion Therapeutics, Immune Pharmaceuticals, Celgene, Arena Pharmaceuticals, Ambrx Inc., Akros Pharma, Vascular Biogenics, Theradiag, Forward Pharma, Regeneron, Galapagos, Seres Health, Ritter Pharmaceuticals, Theravance, Palatin, Biogen Idec, and the University of Western Ontario (owner of Robarts Clinical Trials) outside the submitted work.

\section{REFERENCES}

1. Ng M, Fleming T, Robinson M, et al. Global, regional, and national prevalence of overweight and obesity in children and adults during 1980-2013: a systematic analysis for the Global Burden of Disease Study 2013. Lancet 2014;384:766-81. [PubMed: 24880830]

2. Flores A, Burstein E, Cipher DJ, et al. Obesity in Inflammatory Bowel Disease: A Marker of Less Severe Disease. Dig Dis Sci 2015;60:2436-45. [PubMed: 25799938]

3. Pringle PL, Stewart KO, Peloquin JM, et al. Body Mass Index, Genetic Susceptibility, and Risk of Complications among Individuals with Crohn's Disease. Inflamm Bowel Dis 2015;21:2304-2310. [PubMed: 26181430]

4. Singh S, Dulai PS, Zarrinpar A, et al. Obesity in IBD: epidemiology, pathogenesis, disease course and treatment outcomes. Nat Rev Gastroenterol Hepatol 2017;14:110-121. [PubMed: 27899815]

5. Singh SF M; Shaffer K; Singh AG; Prokop LJ; Grunvald E; Zarrinpar A; Sandborn WJ Pre-Morbid Obesity is Associated with Increased Risk of Developing Immune-Mediated Inflammatory Diseases: a Systematic Review and Meta-Analysis. Gastroenterology 2017;152:S976-S977.

6. Jain AS S; Martin C; Sandler R; Sandborn WJ; Herfarth HH; Kappelman MD; Long MD Obesity is Associated with Worse Disease Activity in Patients with Inflammatory Bowel Diseases: An Internet Based Cohort Study. Gastroenterology 2017;152:S973-S974.

7. Seminerio JL, Koutroubakis IE, Ramos-Rivers C, et al. Impact of Obesity on the Management and Clinical Course of Patients with Inflammatory Bowel Disease. Inflamm Bowel Dis 2015;21:285763. [PubMed: 26241001] 
8. Hojgaard P, Glintborg B, Kristensen LE, et al. The influence of obesity on response to tumour necrosis factor-alpha inhibitors in psoriatic arthritis: results from the DANBIO and ICEBIO registries. Rheumatology 2016;55:2191-2199. [PubMed: 27651526]

9. Iannone F, Fanizzi R, Notarnicola A, et al. Obesity reduces the drug survival of second line biological drugs following a first TNF-alpha inhibitor in rheumatoid arthritis patients. Joint Bone Spine 2015;82:187-91. [PubMed: 25619156]

10. Klaasen R, Wijbrandts CA, Gerlag DM, et al. Body mass index and clinical response to infliximab in rheumatoid arthritis. Arthritis Rheumat 2011;63:359-64. [PubMed: 21279992]

11. Singh SFA, Singh AG, Casteele NV, Zarrinpar A, Grunvald E, Curtis JR, Sandborn WJ. Obesity is Associated with Inferior Response to Anti-TNF Therapy in Immune-Mediated Inflammatory Diseases: A Systematic Review and Meta-Analysis. Gastroenterology 2017;2017:S154.

12. Bhalme M, Sharma A, Keld R, et al. Does weight-adjusted anti-tumour necrosis factor treatment favour obese patients with Crohn's disease? European J Gastroenterol Hepatol 2013;25:543-9. [PubMed: 23337170]

13. Harper JW, Sinanan MN, Zisman TL. Increased body mass index is associated with earlier time to loss of response to infliximab in patients with inflammatory bowel disease. Inflamm Bowel Dis 2013;19:2118-24. [PubMed: 23863401]

14. Passot C, Mulleman D, Bejan-Angoulvant T, et al. The underlying inflammatory chronic disease influences infliximab pharmacokinetics. MAbs 2016;8:1407-1416. [PubMed: 27589009]

15. Wade JR, Parker G, Kosutic G, et al. Population pharmacokinetic analysis of certolizumab pegol in patients with Crohn's disease. J Clin Pharmacol 2015;55:866-74. [PubMed: 25735646]

16. Krumholz HM, Waldstreicher J. The Yale Open Data Access (YODA) Project--A Mechanism for Data Sharing. N Engl J Med 2016;375:403-5. [PubMed: 27518657]

17. Best WR, Becktel JM, Singleton JW, et al. Development of a Crohn's disease activity index. National Cooperative Crohn's Disease Study. Gastroenterology 1976;70:439-44. [PubMed: 1248701]

18. Turner D, Griffiths AM, Walters TD, et al. Appraisal of the pediatric Crohn's disease activity index on four prospectively collected datasets: recommended cutoff values and clinimetric properties. Am J Gastroenterol 2010;105:2085-92. [PubMed: 20372111]

19. Schroeder KW, Tremaine WJ, Ilstrup DM. Coated oral 5-aminosalicylic acid therapy for mildly to moderately active ulcerative colitis. A randomized study. N Engl J Med 1987;317:1625-9. [PubMed: 3317057]

20. R Core Team: A language and environment for statistical computing. R Foundation for Statistical Computing, Vienna, Austria [2013]

21. Ottaviani S, Allanore Y, Tubach F, et al. Body mass index influences the response to infliximab in ankylosing spondylitis. Arthritis Res Ther 2012;14:R115. [PubMed: 22584116]

22. Sheehan AL, Warren BF, Gear MW, et al. Fat-wrapping in Crohn's disease: pathological basis and relevance to surgical practice. Br J Surg 1992;79:955-8. [PubMed: 1422768] 


\section{STUDY HIGHLIGHTS}

\section{What is Current Knowledge?}

- Obesity is associated with increased risk of developing inflammatory bowel disease, and may negatively impact natural history of disease

- Population pharmacokinetic studies suggest that obesity promotes biologic drug clearance

- $\quad$ Studies in patients with rheumatic diseases suggest that obese patients may have inferior response to infliximab and other biologic agents. However, it is unclear whether this holds true in patients with inflammatory bowel diseases

\section{What this study adds?}

- $\quad$ Based on individual participant data from 4 pivotal trials of infliximab in IBD with 1207 patients, we observed no association between obesity and response to infliximab after adjusting for relevant confounders

- $\quad$ These results were stable in trials of induction and maintenance therapy, and in patients with ulcerative colitis and Crohn's disease 


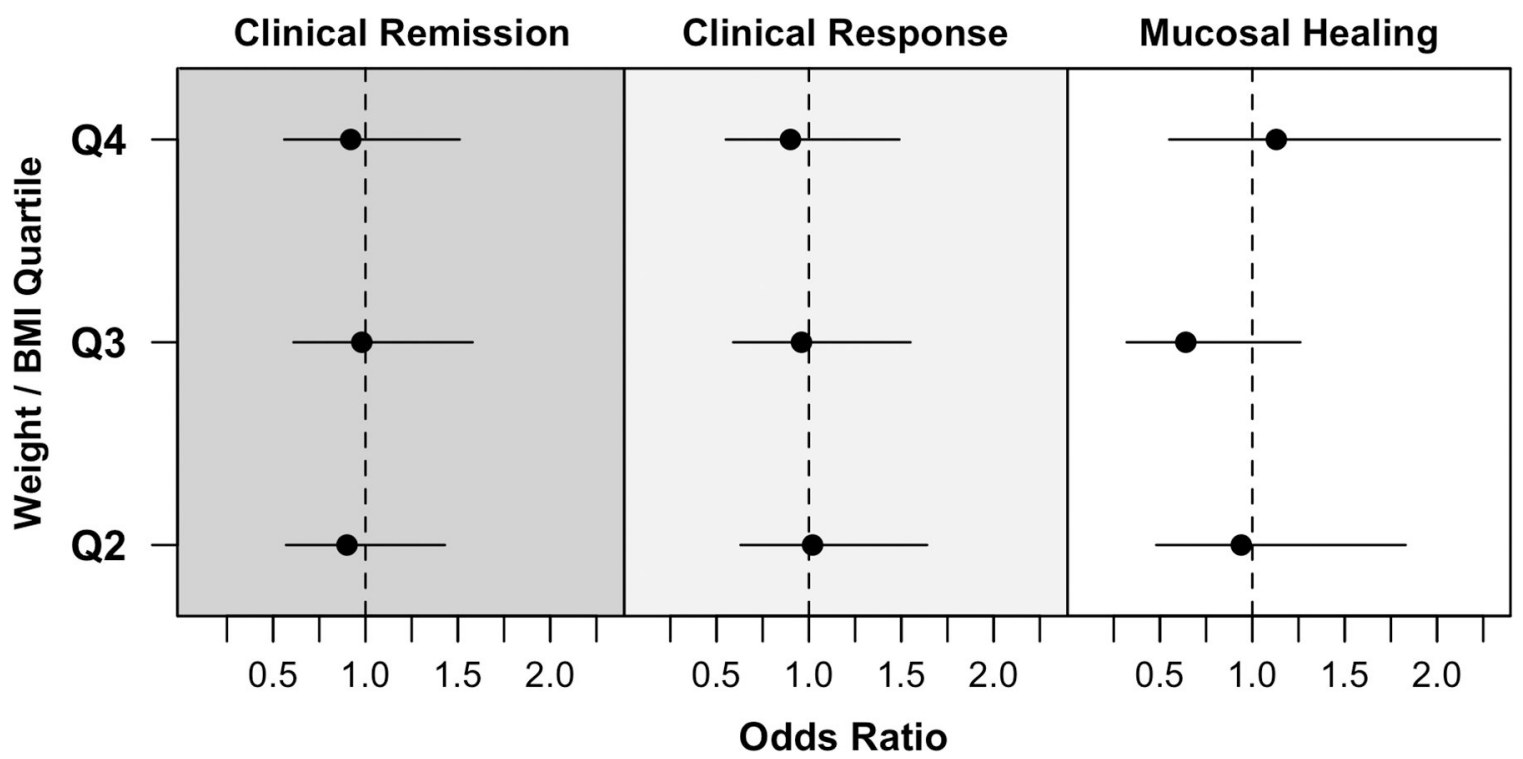

Figure 1.

Clinical remission, clinical response and mucosal healing in infliximab-treated patients across quartiles of $\mathrm{BMI} /$ weight, as compared to quartile 1 as reference. 


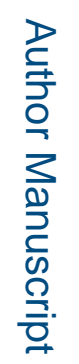

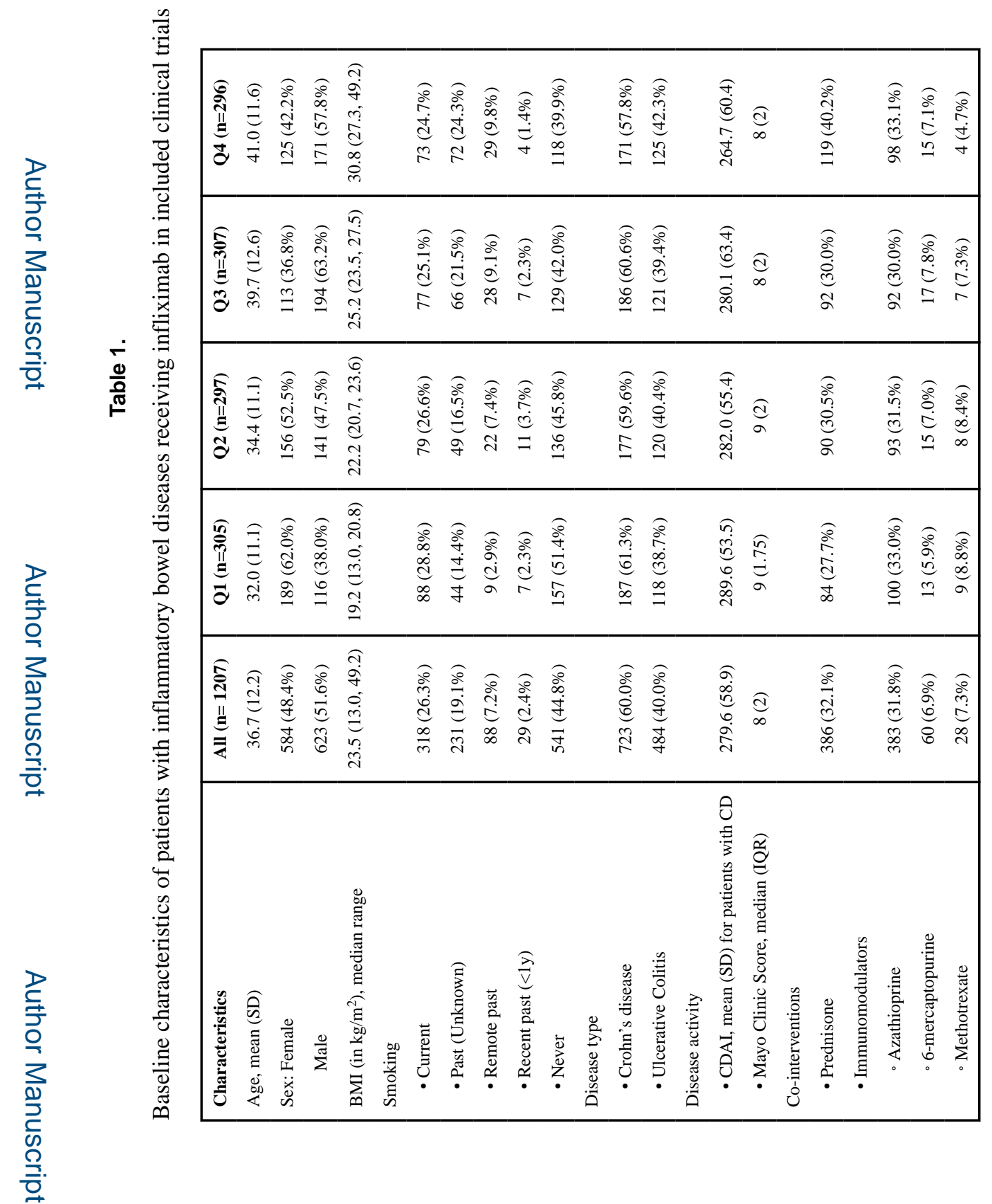

Am J Gastroenterol. Author manuscript; available in PMC 2020 March 31. 


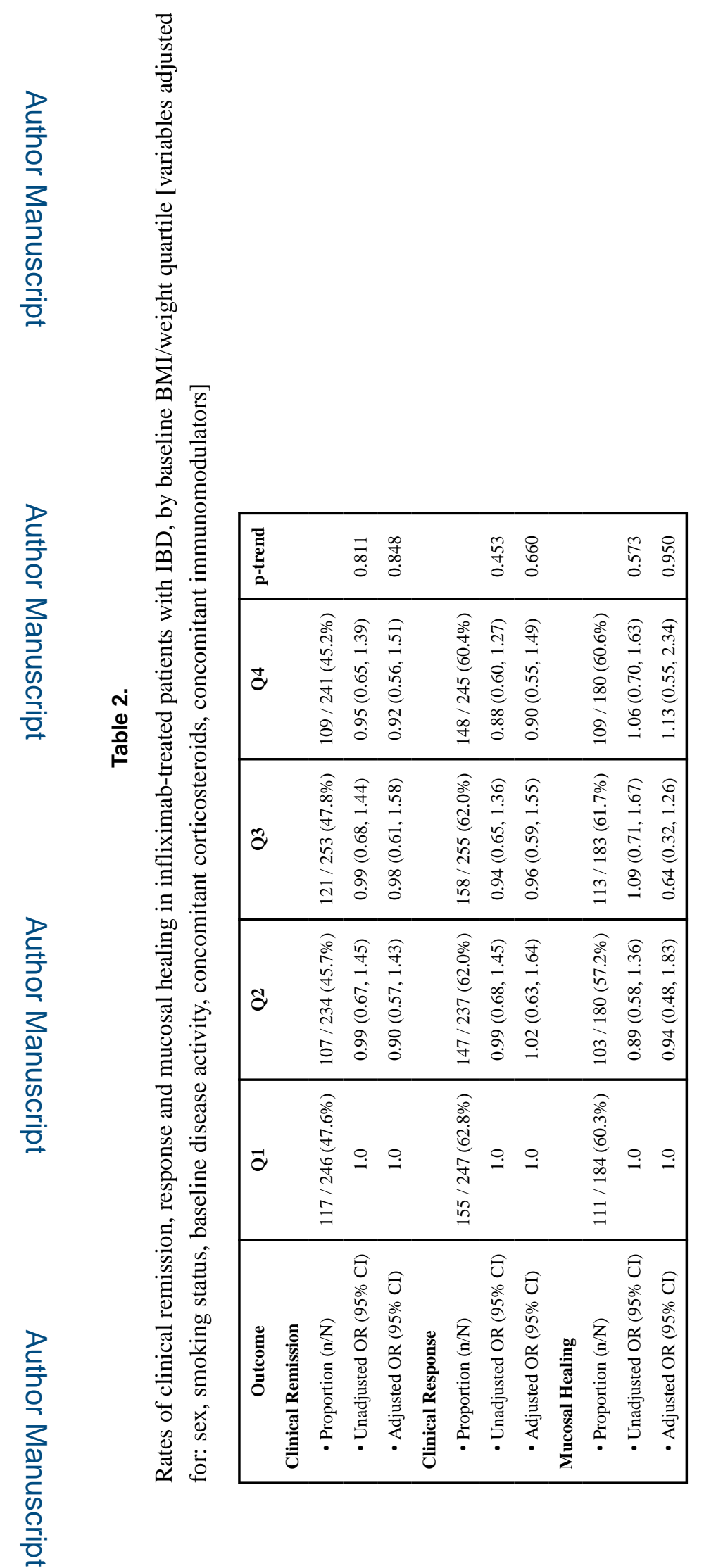

Am J Gastroenterol. Author manuscript; available in PMC 2020 March 31. 


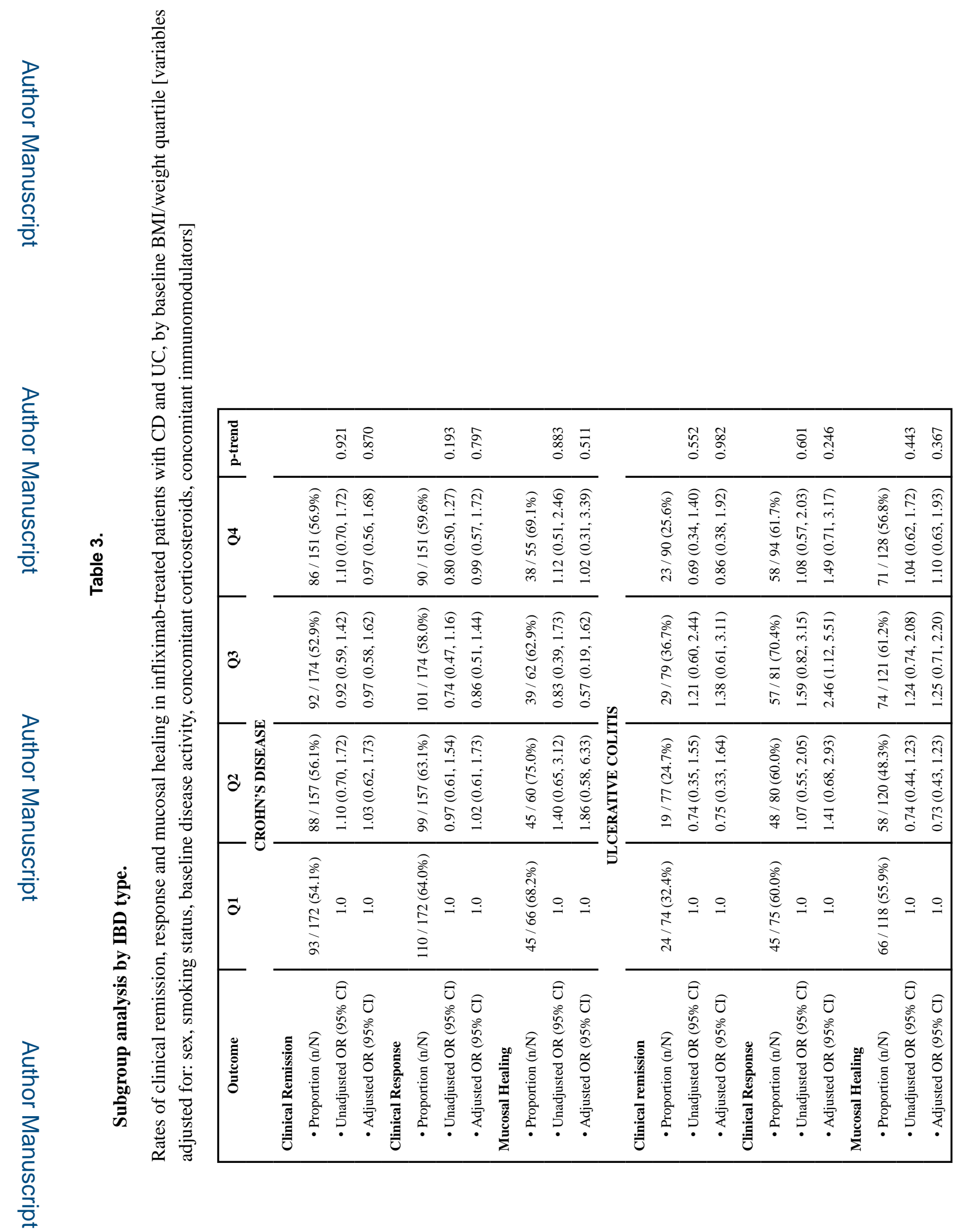

Am J Gastroenterol. Author manuscript; available in PMC 2020 March 31. 


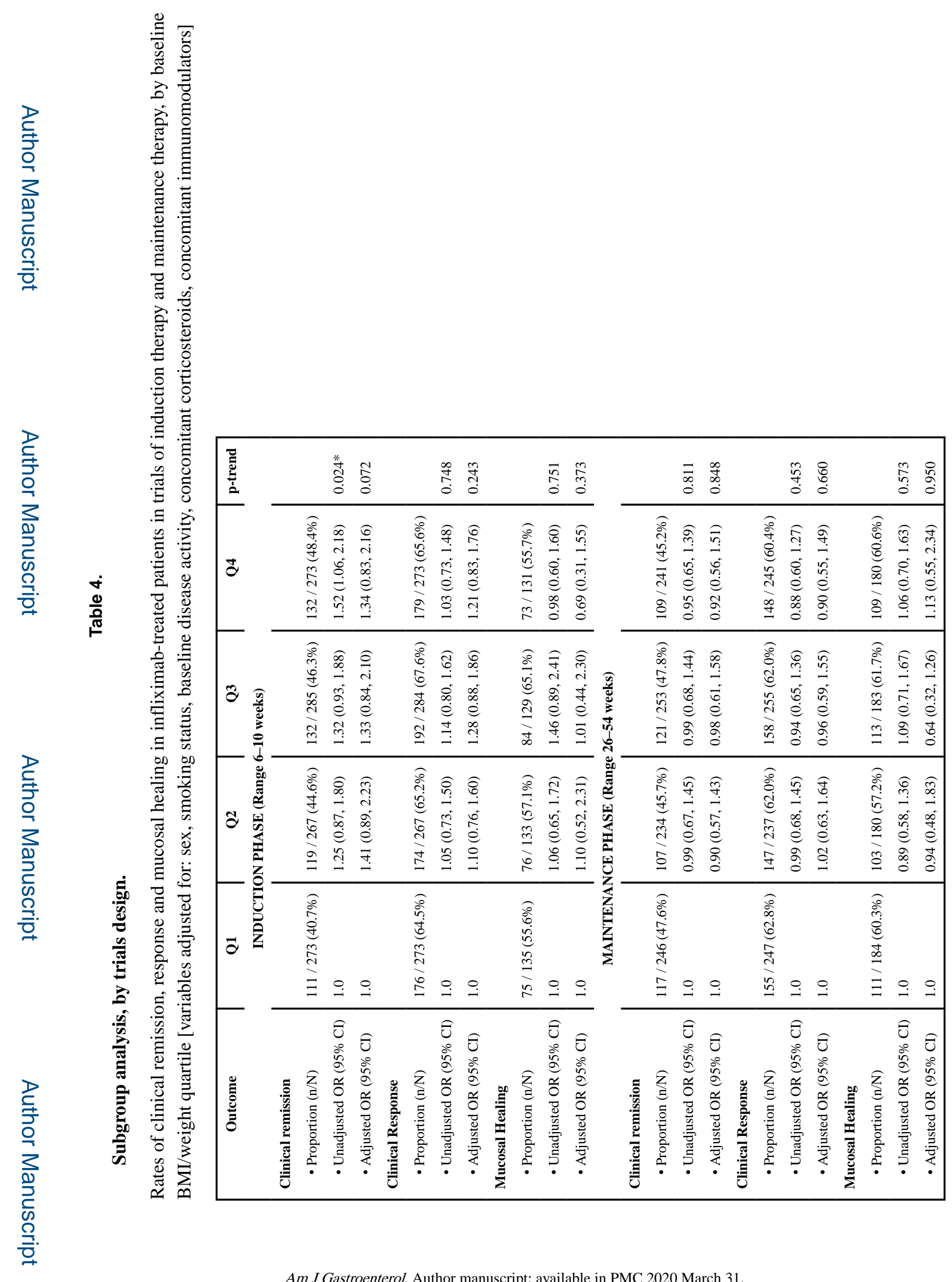

